\title{
SPLITTING FIELD DAN KETUNGGALANNYA ATAS POLINOMIAL FIELD
}

\section{Corina Karim ${ }^{1}$ dan Ari Andari ${ }^{2}$}

1,2Jurusan Matematikam Universitas Brawijaya, Malang

\begin{abstract}
ABSTRAK
Suatu field $E$ disebut extension field $F$, jika $F \subset E$ di mana $F$ merupakan field. Dengan kata lain $E$ disebut extension field $F$, jika $F$ subfield dari field $E$. Sedangkan Splitting field merupakan generalisasi dari extension field yang memenuhi beberapa aksioma. Field yang digunakan pada splitting field adalah field finite extension, dimana field finite extension adalah extension field yang mempunyai basis berhingga $n$.
\end{abstract}

Kata kunci: extension field, finite extension dan splitting field.

\section{PENDAHULUAN}

$R$ ring, suatu barisan tak hingga $\left(a_{0}, a_{1}, a_{2}, \ldots\right) \in R$ dikatakan suatu polinomial atas ring $R$ jika terdapat suatu bilangan bulat tak negatif $n$ sedemikian sehingga terdapat $a_{i}=0 \in R, \forall i>n$. Polinomial tersebut dinotasikan sebagai berikut:

$$
\begin{aligned}
f(x) & =a_{0}+a_{1} x+a_{2} x^{2}+\cdots+a_{n} x^{n}+\cdots \\
& =\sum_{i=1}^{\infty} a_{i} x^{i}
\end{aligned}
$$

dimana $x$ disebut indeterminate atas ring $\mathrm{R}$.

Jika $F$ field dan $F[x]$ ring polinomial $x$ atas $F$ maka $F[x]$ daerah integral dengan elemen kesatuan dan memuat subring sejati $F$. Polinomial $f(x) \in F[x]$ disebut irreducible jika degree $f(x) \geq 1$, dan jika $f(x)=g(x) . h(x)$ dimana $g(x), h(x) \in F[x]$, maka $g(x) \in F$ atau $h(x) \in F$. Jika polinomial $f(x)$ tidak irreducible maka disebut reducible (Bhattacharya, dkk, 1986).

Selanjutnya Fraleigh (1994) menyebutkan bahwa suatu field $E$ disebut extension field $F$, jika $F \subset E$ di mana $F$ merupakan field. Dengan kata lain $E$ disebut extension field $F$, jika $F$ subfield dari field $E$. Dan jika $E$ extension field $F$ yang mempunyai basis berhingga $n$ di mana $E$ adalah ruang vektor atas $F$, maka $E$ adalah suatu finite extension berderajat $n$. Selanjutnya dinotasikan $[E: F]=n$, artinya $E$ extension field $F$ berdimensi $n$.

Di lain pihak, menurut Gallian (1990), $E$ extension field $F$ dan $\alpha \in E, \alpha$ disebut algebraic atas $\mathrm{F}$, jika $\alpha$ akar dari suatu polinomial $\mathrm{F}[x]$. $(f(x) \in F[x], f(\alpha)=0)$. Jika $\alpha$ bukan algebraic atas F, maka $\alpha$ disebut transendental atas F. Dan menurut Fraleigh (1994) suatu $E$ extension field dari field $F$ disebut algebraic extension dari $F$ jika setiap elemen di $E$ algebraic atas $F$. Jika $E$ bukan algebraic extension maka $E$ disebut transcenddental extension.

Termotivasi dari pengertian extension field dan finite extension, maka dalam makalah ini akan dibahas tentang splitting field atas polinomial di $\mathrm{F}(\mathrm{x})$ dan membuktikan ketunggalan dari splitting field tersebut.

\section{PEMBAHASAN}

Definisi 1 (Bhattacharya, dkk, 1986).

Jika $f(x)$ polinomial di $F[x]$ dengan degree $\geq 1$ maka $K$ extension field $\mathrm{F}$ disebut Splitting field $f(x)$ atas $F$ jika:

i) Faktor $f(x)$ dapat ditulis menjadi faktor linier $K[x] \ni$

$f(x)=c\left(x-\alpha_{1}\right)\left(x-\alpha_{2}\right) \ldots\left(x-\alpha_{n}\right), \alpha_{i} \in K$, dengan $c$ sebarang skalar.

ii) $K=F\left(\alpha_{1}, \alpha_{2}, \ldots, \alpha_{n}\right) \ni K$ dibangun oleh $F$ dengan akar-akar $\alpha_{1}, \alpha_{2}, \ldots, \alpha_{n} \in f(x)$ dan $f(x) \in K$.

\section{Contoh 1:}

Field $\mathbb{Q}(\sqrt{2})=\{a+b \sqrt{2} \mid a, b \in \mathbb{Q}\}$ adalah splitting field dari $x^{2}-2 \in \mathbb{Q}[x]$ atas $\mathbb{Q}$.

Bukti:

Jelas $\mathbb{Q} \subseteq \mathbb{Q}(\sqrt{2})$.

Akan dibuktikan:

(i). $\mathbb{Q}(\sqrt{2})$ extension field $\mathbb{Q}$.

(ii). $\mathbb{Q}(\sqrt{2})$ splitting field $\mathbb{Q}[\mathrm{x}]$ atas $\mathbb{Q}$.

Bukti (i).

$\mathbb{Q} \subset \mathbb{Q}(\sqrt{2})$.

Klaim $S=\{1, \sqrt{2}\}$

Akan ditunjukkan:

$S$ adalah basis untuk ruang vektor $\mathbb{Q}(\sqrt{2})$

a) Ambil sebarang $y \in \mathbb{Q}(\sqrt{2})$.

Maka $y$ dapat dinyatakan sebagai

$$
\begin{aligned}
y & =a+b \sqrt{2}, a, b \in \mathbb{Q} \\
& =a \cdot 1+b . \sqrt{2},
\end{aligned}
$$


Jadi S merentang $\mathbb{Q}(\sqrt{2})$.

b) Untuk suatu $a, b \in \mathbb{Q}$, maka

$$
\begin{aligned}
0 & =a+b \sqrt{2} \\
\Rightarrow 0 & =a \cdot 1+b \cdot \sqrt{2}
\end{aligned}
$$

Akan terpenuhi jika $a=0$ dan $b=0$

Jadi S bebas Linier.

Dari a) dan b) terbukti S basis untuk $\mathbb{Q}(\sqrt{2})$ dan $[\mathbb{Q}(\sqrt{2}): \mathbb{Q}]=2$. Jadi $\mathbb{Q}(\sqrt{2})$ finite extension $\mathbb{Q}$. Karena $\mathbb{Q}(\sqrt{2})$ finite extension $\mathbb{Q}$, maka $\mathbb{Q}(\sqrt{2})$ extension field $\mathbb{Q}$.

Bukti (ii).

Akan ditunjukkan:

a) $g(x)=c\left(x-\alpha_{1}\right)\left(x-\alpha_{2}\right) \ldots\left(x-\alpha_{n}\right)$

$$
\alpha_{i} \in \mathbb{Q}(\sqrt{2})
$$

Pilih $g(x)=x^{2}-2 \in \mathbb{Q}[x]$

$$
\begin{aligned}
g(x) & =x^{2}-2 \\
& =(x-\sqrt{2})(x+\sqrt{2}) \\
& =(x-\sqrt{2})(x-(-\sqrt{2}))
\end{aligned}
$$

di mana $\pm \sqrt{2} \in \mathbb{Q}(\sqrt{2})$

b) $\mathbb{Q}(\sqrt{2})=\mathbb{Q}\left(\alpha_{1}, \alpha_{2}, \ldots, \alpha_{n}\right) \ni \mathbb{Q}(\sqrt{2})$ dibangun oleh $\mathbb{Q}$ dengan akar-akar $\alpha_{1}, \alpha_{2}, \ldots, \alpha_{n} \in g(x)$ atas $\mathbb{Q}$.

Karena $\mathbb{Q}(\sqrt{2})$ finite extension atas $\mathbb{Q}$ maka $\mathbb{Q}(\sqrt{2})$ algebraic extension atas $\mathbb{Q}$. Sehingga $\exists$ $g(x) \in \mathbb{Q}[x], g(x) \neq 0$ di mana $g(\alpha)=0$.

Ambil sebarang $\alpha \in \mathbb{Q}(\sqrt{2})$ maka

$$
\begin{aligned}
& \alpha=a+\sqrt{2} b \\
& \alpha^{2}=(a+\sqrt{2} b)^{2} \\
& \quad=a^{2}+2 \sqrt{2} a b+2 b^{2} \\
& \alpha^{2}-a^{2}+2 \sqrt{2} a b+2 b^{2}=0
\end{aligned}
$$

Sehingga $\exists g(x) \in \mathbb{Q}[x]$ di mana

$$
g(x)=x^{2}-a^{2}+2 \sqrt{2} a b+2 b^{2}
$$

Jadi $\mathbb{Q}(\sqrt{2})$ dibangun oleh $\mathbb{Q}$ dengan $\alpha \in \mathbb{Q}[x]$

Jadi $\mathbb{Q}(\sqrt{2})$ splitting field $g(x)$ atas $\mathbb{Q}$.

\section{Contoh 2}

Splitting field $x^{2}+1 \in \mathbb{C}[x]$ atas $\mathbb{C}$ adalah field $\mathbb{C}$.

Teorema 2 (Bhattacharya, dkk,1986)

Jika $\mathrm{K}$ splitting field $f(x) \in F[x]$ atas $F$ maka $\mathrm{K}$ finite extension $F$ dan $\mathrm{K}$ algebraic extension atas $F$.

\section{Bukti :}

Karena menurut definisi 1 . bagian ii) $\mathrm{K}=$ $F\left(\alpha_{1}, \alpha_{2}, \ldots, \alpha_{n}\right) \ni \mathrm{K}$ dibangun oleh $F$ dengan $\alpha_{1}, \alpha_{2}, \ldots, \alpha_{n} \in K$. Maka $\mathrm{K}$ algebraic atas $\mathrm{F}$.

Sehingga $\mathrm{K}$ finite extension $F$.

Karena $\mathrm{K}$ finite extension maka $\mathrm{K}$ juga algebraic extension $\mathrm{F}$.
Teorema 3 (Dummit and Foote, 1991)

Misal $\varphi: F \stackrel{\sim}{\longrightarrow} F^{\prime}$ Isomorfisma Field.

$f(x) \in F[x]$ polinomial dan $f^{\prime}(x) \in F^{\prime}[x]$ polinomial yang didapat dari $\varphi$ terhadap koefisien $f(x)$. Jika $E$ splitting field $f(x)$ atas $F$ dan $E^{\prime}$ splitting field $f^{\prime}(x)$ atas $F^{\prime}$. Maka Isomorfisma $\varphi$ "extended to" isomorfisma $\sigma: E \stackrel{\sim}{\longrightarrow} E^{\prime}, \sigma$ yang dibatasi (restricted to) $F$ adalah Isomorfisma $\varphi$ :

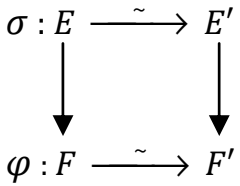

Bukti:

$\varphi: F \stackrel{\sim}{\longrightarrow} F^{\prime}$ isomorfisma Field.

$f(x) \mapsto f^{\prime}(x)$

$\alpha_{0}+\alpha_{1} x \mapsto \alpha_{0}{ }^{\prime}+\alpha_{1}{ }^{\prime} x$

E splitting field $f(x)$ atas $F$

$E^{\prime}$ splitting field $f^{\prime}(x)$ atas $F^{\prime}$

Akan dibuktikan:

a)

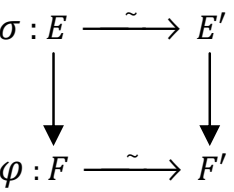

b) $\sigma$ Isomorfisma

Bukti:

a) Dengan menggunakan induksi matematika terhadap derajat $n$ pada $E$ field extension $F$.

i) Untuk $n=1$.

Jika $n=1$ maka $E=F$

Padahal

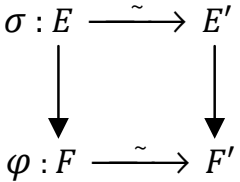

maka $\sigma=\varphi$.

ii) Andai untuk $n=2$ benar maka akan dibuktikan $n>2$ benar untuk sebarang field extension.

Bukti:

Definisikan : $\phi: F(x) \rightarrow F(\alpha)$

$$
p \mapsto p(\alpha)
$$

Ambil sebarang $\alpha \in E$ splitting field $f(x), f(x) \notin F$.

Misal $m(x)$ polinomial terkecil yang memuat $\alpha$. maka $m(x) \mid f(x)$, karena $\alpha \in f(x)$. 
Ambil sebarang $m^{\prime(x)} \in F^{\prime}[x] \ni m(x)=$ $m^{\prime}(x)$.

Karena $f^{\prime}(x)$ splitting field atas $F^{\prime}$ maka ada elemen $\beta \in F^{\prime}$ dan $\beta \in m^{\prime}(x)$.

Jadi ada homomorfisma ring $\pi$ extending $\varphi \ni$

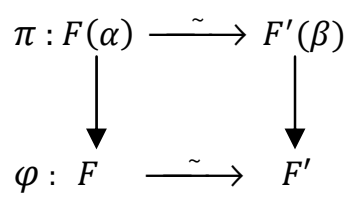

karena $[F(\alpha): F]>1$ maka:

$[F(\alpha): F] \geq 2$

$[E: F(\alpha)][F(\alpha): F] \geq[E: F(\alpha)] .2$

$[E: F] \geq[E: F(\alpha)] .2 \quad$ (karena $[E: F]=2$ benar)

maka $[E: F(\alpha)] \leq 1$

Jadi $[E: F(\alpha)]<1$ dan $[E: F(\alpha)]=1$.

Jika $[E: F(\alpha)]<1$ maka ada $\sigma$ extending $\pi \ni$

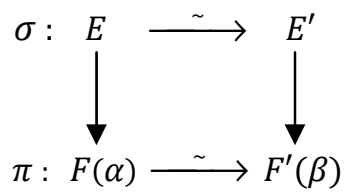

karena $\sigma$ extends $\pi$ dan $\pi$ extends $\varphi$ maka $\sigma$ extends $\varphi$.

Dari (1) dan (2) diperoleh:

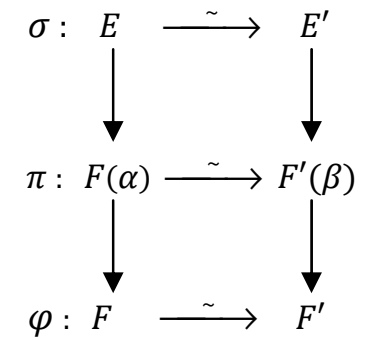

Jadi

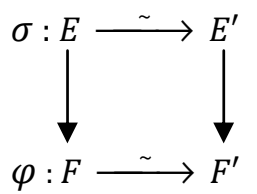

Jika $[E: F(\alpha)]=1$ maka ada $\sigma$ extending $\pi \ni$

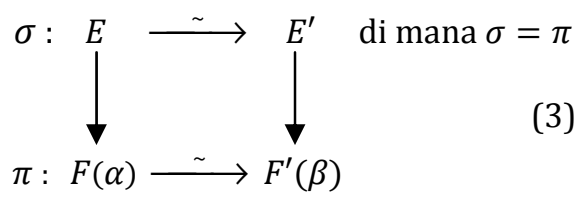

Dari (1) dan (3) diperoleh

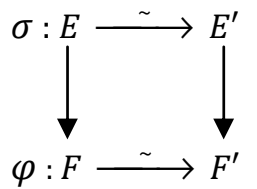

b) Andai $E^{\prime}$ splitting field $f^{\prime}(x)$ atas $F^{\prime}$ dan $\varphi$ isomorfisma.

Akan dibuktikan:

$\sigma$ isomorfisma $\Rightarrow$ i) $\sigma$ homomorfisma

ii) $\sigma$ onto

iii) $\sigma$ satu-satu

Bukti:

i) Didefinisikan:

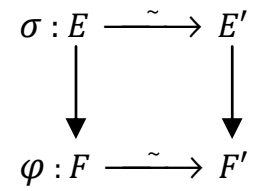

$$
\begin{aligned}
& \sigma(E)=E^{\prime} \\
& \ni \\
& \varphi(F)=F^{\prime}
\end{aligned}
$$

Ambil sebarang $\alpha, \beta \in E$ splitting field $f(x)$ atas $F$ dan $k \in \mathbb{Z}$, maka:

$$
\begin{aligned}
\sigma(\alpha \beta) & =(\alpha \beta)^{\prime} \\
& =\alpha^{\prime} \beta^{\prime} \\
& =\sigma(\alpha) \cdot \sigma(\beta) \\
-\sigma(k \alpha) & =(k \alpha)^{\prime} \\
& =k \cdot \alpha^{\prime} \\
& =k \cdot \sigma(\alpha)
\end{aligned}
$$

Jadi $\sigma$ homomorfisma.

ii) Ambil sebarang $f(x) \in F[x]$, maka:

$f(x)=\alpha_{0}+\alpha_{1} x+\cdots+\alpha_{n} x^{n}, \alpha_{i} \in F$

Himpunan

$f^{\varphi}(x)=\varphi\left(\alpha_{0}+\alpha_{1} x+\cdots+\alpha_{n} x^{n}\right)$

karena $\varphi$ homomorfisma, maka:

$$
\begin{aligned}
f^{\varphi}(x) & =\varphi\left(\alpha_{0}\right)+\varphi\left(\alpha_{1} x\right)+\cdots+\varphi\left(\alpha_{n} x^{n}\right) \\
& =\varphi\left(\alpha_{0}\right)+\varphi\left(\alpha_{1}\right) x+\cdots+\varphi\left(\alpha_{n}\right) x^{n}
\end{aligned}
$$

Padahal $E^{\prime}$ splitting field $f^{\prime}(x) \in F^{\prime}(x)$, maka:

$f^{\prime}(x)=c\left(x-\alpha_{1}\right)\left(x-\alpha_{2}\right) \ldots\left(x-\alpha_{n}\right)$

di mana $\alpha_{i} \notin F^{\prime}$

Sehingga

$$
\begin{aligned}
f^{\prime}(x)= & c\left(x-\varphi\left(a_{1}\right)\right)\left(x-\varphi\left(a_{2}\right)\right) \ldots \\
& \left(x-\varphi\left(a_{n}\right)\right)
\end{aligned}
$$

Disisi lain, jika ada $\beta_{i} \in F^{\prime}$, maka:

$f^{\prime}(x)=c\left(x-\beta_{1}\right)\left(x-\beta_{2}\right) \ldots\left(x-\beta_{n}\right)$

dari (4) dan (5) maka himpunan

$\left\{\varphi\left(a_{1}\right), \varphi\left(a_{2}\right), \ldots, \varphi\left(a_{n}\right)\right\}=\left\{\beta_{1}, \beta_{2}, \ldots, \beta_{n}\right\}$

Karena $E^{\prime}$ splitting field $f^{\prime}(x) \in F^{\prime}(x)$ maka

$$
\begin{aligned}
E^{\prime} & =F^{\prime}\left(\beta_{1}, \beta_{2}, \ldots, \beta_{n}\right) \\
& =F^{\prime}\left(\varphi\left(a_{1}\right), \varphi\left(a_{2}\right), \ldots, \varphi\left(a_{n}\right)\right) \\
& =F^{\prime}\left(\sigma\left(a_{1}\right), \sigma\left(a_{2}\right), \ldots, \sigma\left(a_{n}\right)\right) \\
& =\varphi(F)\left(\sigma\left(a_{1}\right), \sigma\left(a_{2}\right), \ldots, \sigma\left(a_{n}\right)\right) \\
& =\sigma(F)\left(\varphi\left(a_{1}\right), \varphi\left(a_{2}\right), \ldots, \varphi\left(a_{n}\right)\right) \\
& =\sigma\left(F\left(\alpha_{1}, \alpha_{2}, \ldots, \alpha_{n}\right)\right)
\end{aligned}
$$


$E^{\prime}=\sigma(E)$

Jadi $\sigma$ Onto.

iii) $\sigma$ Satu-satu.

Ambil sebarang $x_{1}, x_{1} \in E^{\prime} \exists$

$$
\begin{aligned}
& \quad \sigma\left(x_{1}\right)=\sigma\left(x_{2}\right) \\
& \sigma\left(x_{1}\right)=\sigma\left(x_{2}\right) \\
& \sigma\left(x_{1}\right)-\sigma\left(x_{2}\right)=0 \\
& \sigma\left(x_{1}-x_{2}\right)=0
\end{aligned}
$$

(karena $\sigma$ homomorfisma)

Jadi $x_{1}-x_{2} \in \operatorname{ker} \sigma$

$\sigma\left(x_{1}\right)=\sigma\left(x_{2}\right)$

$\sigma\left(x_{1}\right)-\sigma\left(x_{2}\right)=0$

$\varphi\left(x_{1}\right)-\varphi\left(x_{2}\right)=0$

$\varphi\left(x_{1}-x_{2}\right)=0$

$($ karena $\varphi=\sigma$ )

(karena $\varphi$ homomorfisma)

Jadi $x_{1}-x_{2} \in \operatorname{ker} \varphi$

Jadi $\sigma$ Satu-satu.

\section{Akibat 4 (Ketunggalan Splitting Field)}

Sebarang dua splitting field polinomial $f(x) \in F[x]$ atas field $F$ adalah isomorfik.

Bukti:

Ambil sebarang splitting field $f(x) \in F[x]$.

Misal: E splitting field $f(x)$ atas $F$ dan

$E^{\prime}$ splitting field $f^{\prime}(x)$ atas $F^{\prime}$.

E splitting field $f(x) \in F[x]$ isomorfik dengan $E^{\prime}$ splitting field $f^{\prime}(x) \in F^{\prime}[x]$ jika ada isomorfisma ring

$\sigma: E \rightarrow E^{\prime}$ dan $\varphi: F \rightarrow F^{\prime}$

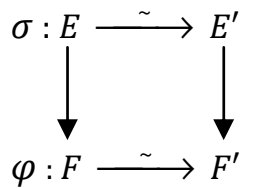

komutatif $(\sigma=\varphi)$.

Jelas. Dari teorema 3 , di mana $F=F^{\prime}$ dan $\sigma \cong \varphi$.

\section{KESIMPULAN}

Dari hasil pembahasan dapat ditarik kesimpulan bahwa jika E dan E' splitting field atas polinomial-polinomial $\mathrm{f}(\mathrm{x}) \in \mathrm{F}[\mathrm{x}]$ maka kedua splitting field tersebut isomorfik. Dan untuk selanjutnya disebut ketunggalan splitting field.

\section{DAFTAR PUSTAKA}

[1] Anton,H. 1987. Aljabar Linier Elementer. Erlangga. Jakarta.

[2] Arifin, A. 2000. Aljabar. ITB. Bandung.

[3] Bhattacharya, P.B., Jain, S.K., Nagpaul, S.R. 1986. Basic Abstract Algebra. Second Ed., Cambridge University Press.USA.

[4] Birkhoff, G., and Mac Lane, S. 1953. A Survey of Modern Algebra. Mac Millan Company. New York.

[5] Deieker, P.F., and Voxman.1986. Discrete Mathematics. Harcourt Brace Jovanovich, Inc. New York.

[6] Dummit, David S., and Foote, Richard M. 1991. Abstract Algebra. Prenticehall, Inc. New Jersey.

[7] Durbin, J.R. 1992. Modern Algebra and Introduction. John Wiley and Sons, Inc. New york.

[8] Fraleigh, John B. 1994. A First Course in Abstract Algebra. Fifth Ed., Addison Wesley Publishing Company, Inc. USA.

[9] Gallian, J.A. 1990. Contemporary Abstract Algebra. DC Heath and Company. USA.

[10] Herstein, I.N. 1975. Topics in Algebra. Second Ed., John Wiley and Sons. Singapore.

[11] Hartley, B., and Hawkes, T.0. 1970. Rings, Modules, and Linear Algebra. Chapman and Hall. London.

[12] Leon, Steven J. 2001. Aljabar Linear dan Aplikasinya. Edisi kelima. Erlangga. Jakarta.

[13] Raisinghania, M.D., and Anggarwal, R.S. 1980. Modern Algebra. S.Chard and Company LTD. New Delhi.

[14] Roman, S. 1991. Advanced Linear Algebra. Springer Verlag. New York.

[15] Sims, Charles C. 1984. Abstract A Computational Approach. John Wiley and Sons, Inc. New York.

[16] Spindler, Karlheins. 1994. Abstract Algebra with Application. Volume II. Marcell Dekker, Inc. USA.

[17] Stewart, Ian. 1989. Galois Theory. Second Ed., Chapman and Hall. London.

[18] Whitelaw, Thomas A. 1995. Introduction to Abstract Algebra. Third Ed., Chapman and Hall. New York. 